\title{
The Relativistic Quantum Motions.
}

\author{
T. Djama*
}

July 1, 2004

\begin{abstract}
Using the relativistic quantum stationary Hamilton-Jacobi equation within the framework of the equivalence postulate, and grounding oneself on both relativistic and quantum Lagrangians, we construct a Lagrangian of a relativistic quantum system in one dimension and derive a third order equation of motion representing a first integral of the relativistic quantum Newton's law. Then, we plot the relativistic quantum trajectories of a particle moving under the constant and the linear potentials. We establish the existence of nodes and link them to the de Broglie's wavelength.
\end{abstract}

PACS: 03.65.Bz; 03.65.Ca;

Key words: relativistic quantum law of motion, Lagrangian, relativistic quantum stationary Hamilton-Jacobi equation, relativistic velocities.

${ }^{*}$ E-mail: djam_touf@yahoo.fr 


\section{Introduction}

Deriving quantum mechanics from an equivalence postulate, Faraggi and Matone showed that the Schrödinger wave function must have the form [1, 2, 3, 4]

$$
\phi(x)=\left(\frac{\partial S_{0}}{\partial x}\right)^{-\frac{1}{2}}\left[\alpha \exp \left(\frac{i}{\hbar} S_{0}\right)+\beta \exp \left(-\frac{i}{\hbar} S_{0}\right)\right],
$$

where $\alpha$ and $\beta$ are complex constants and $S_{0}$ is a real function representing the quantum reduced action. They established that the conjugate momentum given by

$$
P=\frac{\partial S_{0}}{\partial x}
$$

never vanishes for bound and unbound states making possible a dynamical approach of the quantum motion of particles. This conjugate momentum is always real even in classically forbidden regions. They showed also, within the framework of differential geometry [1, 2, 3, 4, that the quantum stationary Hamilton-Jacobi equation (QSHJE) which leads to the Shrödinger equation is

$\frac{1}{2 m_{0}}\left(\frac{\partial S_{0}}{\partial x}\right)^{2}+V(x)-E=\frac{\hbar^{2}}{4 m_{0}}\left[\frac{3}{2}\left(\frac{\partial S_{0}}{\partial x}\right)^{-2}\left(\frac{\partial^{2} S_{0}}{\partial x^{2}}\right)^{2}-\left(\frac{\partial S_{0}}{\partial x}\right)^{-1}\left(\frac{\partial^{3} S_{0}}{\partial x^{3}}\right)\right]$

where $V(x)$ is the potential and $E$ the energy. The solution of Eq. (3) investigated by Floyd [5, 6, 7, 8, and Faraggi and Matone [1, 2, 3, 4, is given in Ref. [9] as

$$
S_{0}=\hbar \arctan \left(a \frac{\theta}{\phi}+b\right)
$$

where $a$ and $b$ are real constants. $\theta$ and $\phi$ are two real independent solutions 
of the Schrödinger equation. Taking advantage on these results, Bouda and Djama have recently introduced a quantum Lagrangian

$$
L(x, \dot{x}, \mu, \nu)=\frac{1}{2} m \dot{x}^{2} f(x, \mu, \nu)-V(x)
$$

from which they derived the quantum law of motion [9]. They stated that the conjugate momentum of the non relativistic and spinless particle is written as

$$
\dot{x} \frac{\partial S_{0}}{\partial x}=2(E-V)
$$

From this last equation, they derived the first integral of the quantum Newton's law (FIQNL).

$$
\begin{aligned}
(E-V)^{4}-\frac{m \dot{x}^{2}}{2}(E-V)^{3}+\frac{\hbar^{2}}{8}\left[\frac{3}{2}\left(\frac{\ddot{x}}{\dot{x}}\right)^{2}-\frac{\dot{x}}{\dot{x}}\right](E-V)^{2} & \\
- & \frac{\hbar^{2}}{8}\left[\dot{x}^{2} \frac{d^{2} V}{d x^{2}}+\ddot{x} \frac{d V}{d x}\right](E-V)-\frac{3 \hbar^{2}}{16}\left[\dot{x} \frac{d V}{d x}\right]^{2}=0,
\end{aligned}
$$

which goes at the classical limit $(\hbar \rightarrow 0)$ to the classical conservation equation

$$
\frac{m \dot{x}^{2}}{2}+V(x)=E .
$$

Bouda and Djama have also plotted some trajectories of the particle for several potentials [10].

The construction of the Lagrangian (5) and the establishment of the fundamental dynamical equations (6) and (7) are important steps to build a deterministic theory which restores the existence of trajectories [9, 10, 11] . Nevertheless, such a formalism cannot approach both relativistic velocities cases, and more than one dimension motions. The aim of this paper is to 
generalize the dynamical formalism that we have recalled above [9, 10, 11] into the one dimensional relativistic velocities cases. In this purpose let us recall the finding of Faraggi, Matone and Bertoldi concerning the relativistic quantum systems. They stated that the relativistic quantum wave function is given by Eq. (1), where $S_{0}$ defines the relativistic quantum reduced action, and wrote the relativistic quantum stationary Hamilton-Jacobi equation (RQSHJ) as [3, 4]

$$
\begin{array}{r}
\frac{1}{2 m_{0}}\left(\frac{\partial S_{0}}{\partial x}\right)^{2}-\frac{\hbar^{2}}{4 m_{0}}\left[\frac{3}{2}\left(\frac{\partial S_{0}}{\partial x}\right)^{-2}\left(\frac{\partial^{2} S_{0}}{\partial x^{2}}\right)^{2}-\right. \\
\left.\left(\frac{\partial S_{0}}{\partial x}\right)^{-1}\left(\frac{\partial^{3} S_{0}}{\partial x^{3}}\right)\right]+\frac{1}{2 m_{0} c^{2}}\left[m_{0}^{2} c^{4}-(E-V)^{2}\right]=0
\end{array}
$$

where $V$ is the potential, $E$ is the total energy of the particle of mass equal to $m_{0}$ at rest and $c$ is the light velocity in vacuum. The solution of Eq. (9) can be expressed by Eq. (4), where $\theta$ and $\phi$ represent now two real independent solutions of the Klein-Gordon equation

$$
-c^{2} \hbar^{2} \frac{\partial^{2} \phi}{\partial x^{2}}+\left[m_{0}^{2} c^{4}-(E-V)^{2}\right] \phi(x)=0 .
$$

Taking advantage on these results, we will introduce in the following sections a relativistic quantum formalism with which we study the dynamics of high energy particles. First, in Sec. 2, we present a relativistic quantum Lagrangian from which we derive the relativistic quantum law of motion. Then, in Sec. 3 we study and plot the relativistic quantum trajectories (RQTs) of a particle moving under the constant potential. In Sec. 4, we study the linear potential case. Finally, in Sec. 5, we introduce our definition of de Broglie's wavelength. 


\section{The relativistic quantum motion}

Now, we consider a relativistic quantum system. As we have noticed for the quantum systems [9], the relativistic quantum reduced action $S_{0}$ expressed by Eq. (4) contains two constants more than the usual constant $E$ appearing in the expression of the classical reduced action. This suggests that the relativistic quantum law of motion is a fourth order differential equation. Then, as in the quantum case [9], we introduce in the expression of the Lagrangian a function $f$ of $x$ depending on the constants $a$ and $b$ playing the role of hidden parameters. As the relativistic quantum Lagrangian must goes at the classical limit $(\hbar \rightarrow 0)$ to the relativistic one, we postulate the following form for the Lagrangian

$$
L(x, \dot{x}, a, b)=-m_{0} c^{2} \sqrt{1-\frac{\dot{x}^{2}}{c^{2}} f(x, a, b)}-V(x),
$$

in which the function $f(x, a, b)$ satisfies

$$
\lim _{\hbar \rightarrow 0} f(x, a, b)=1
$$

Since $L$ depends only on the variables $x, \dot{x}$ and the constants $a, b$, the conjugate momentum is given by

$$
P=\frac{\partial L}{\partial \dot{x}}=\frac{m_{0} \dot{x} f(x, a, b)}{\sqrt{1-\left(\dot{x}^{2} / c^{2}\right) f(x, a, b)}} .
$$

The Hamiltonian corresponding to the Lagrangian (11) is

$$
H=P \dot{x}-L
$$


Replacing Eqs. (11) and (13) into Eq. (14), one obtains

$$
H(x, P)=\sqrt{m_{0}^{2} c^{4}+\frac{P^{2} c^{2}}{f(x, a, b)}}+V(x),
$$

At the classical limit, we see clearly by using Eq. (12) that the relativistic quantum momentum $P$ given in Eq. (13) reduces to the relativistic one expressed as

$$
P=\frac{m_{0} \dot{x}}{\sqrt{1-\dot{x}^{2} / c^{2}}} .
$$

Likewise, the Hamiltonian given in Eq. (15) reduces to his relativistic form

$$
H(x, P)=\sqrt{m_{0}^{2} c^{4}+P^{2} c^{2}}+V(x),
$$

well known in special relativity. For the stationary cases the Hamiltonian $H$ corresponds to the total energy $E$ of the particle. Then, we can write Eq. (15) as

$$
E=\sqrt{m_{0}^{2} c^{4}+\frac{P^{2} c^{2}}{f(x, a, b)}}+V(x),
$$

which reads

$$
\frac{1}{2 m_{0}}\left(\frac{\partial S_{0}}{\partial x}\right)^{2} \frac{1}{f(x, a, b)}+\frac{1}{2 m_{0} c^{2}}\left[m_{0}^{2} c^{4}-(E-V)^{2}\right]=0,
$$

after taking into account of the Hamilton-Jacobi definition of the conjugate momentum $P=\partial S_{0} / \partial x$. By applying the equivalence postulate, already introduced by Faraggi and Matone in previous works [1, 2, 3, 4, on Eq. (19), we obtain the RQSHJE given in Eq. (9). This result suggests strongly that the introduction of a Lagrangian with the form (11) is founded. 
Now, taking the expression of the function $f(x, a, b)$ from Eq. (13) into Eq. (19), we get

$$
\dot{x} \frac{\partial S_{0}}{\partial x}=E-V(x)-\frac{m_{0}^{2} c^{4}}{(E-V)} .
$$

The last equation represents the relativistic quantum law of motion, so the relativistic quantum trajectories of a particle moving under any potential $V(x)$ should be plotted using Eq. (20). To proceed, one can deduce the expression of the conjugate momentum from Eq. (4) and replace it into Eq. (20), then we can integrate this equation to give the relativistic quantum time equation $x(t)$. Note that at the classical limit $\hbar \rightarrow 0$, Eq. (20) reduces to the relativistic conservation equation

$$
E=\frac{m_{0} c^{2}}{\sqrt{1-\dot{x}^{2} / c^{2}}}+V(x)
$$

since the conjugate momentum takes the form (16). Note also that in the relativistic limit $(c \rightarrow \infty)$, the kinetic energy $T=E-V-m_{0} c^{2}$ satisfies $T \ll m_{0} c^{2}$, so , Eq. (20) reduces to

$$
\dot{x} \frac{\partial S_{0}}{\partial x}=2 T
$$

which is equivalent to Eq. (6) already established in Ref [9].

Now, deducing $\partial^{2} S_{0} / \partial x^{2}$ and $\partial^{3} S_{0} / \partial x^{3}$ from Eq. (20) and replacing them into the expression of the RQSHJE (Eq. (9)), we find the First Integral of the Relativistic Quantum Newton's Law FIRQNL which reads 


$$
\begin{gathered}
{\left[(E-V)^{2}-m_{0}^{2} c^{4}\right]^{3}\left[\left(1-\frac{\dot{x}^{2}}{c^{2}}\right)-\frac{m_{0}^{2} c^{4}}{(E-V)^{2}}\right]} \\
-\frac{\hbar^{2}}{2}\left[\frac{(E-V)^{4}-m_{0}^{4} c^{8}}{E-V}\right]\left(\ddot{x} \frac{d V}{d x}+\dot{x}^{2} \frac{d^{2} V}{d x^{2}}\right) \\
+\frac{\hbar^{2}}{2}\left[(E-V)^{2}-m_{0}^{2} c^{4}\right]^{2}\left[\frac{3}{2}\left(\frac{\ddot{x}}{\dot{x}}\right)^{2}-\frac{\dot{x}}{\dot{x}}\right] \\
-\frac{\hbar^{2}}{4}\left[4 m_{0}^{2} c^{4}\left(1-\frac{m_{0}^{2} c^{4}}{(E-V)^{2}}\right)-\right. \\
\left.3\left(E-V+\frac{m_{0}^{2} c^{4}}{E-V}\right)^{2}\right]\left(\dot{x} \frac{d V}{d x}\right)^{2}=0
\end{gathered}
$$

As we observe, Eq. (22) is a third order differential equation in $x$ containing the first and the second derivatives of the potential $V$ with respect to $x$. Then, its solution $x(t, E, a, b, c)$ contains four integration constants which can be determined by the initial conditions. It is clear that if we set $\hbar=0, \mathrm{Eq}$. (22) reduces to Eq.(21) representing the relativistic conservation equation. Note also that, after taking the relativistic limit $(c \rightarrow \infty)$ into Eq. (22), one gets Eq. (7) representing the FIQNL.

\section{Motion under the constant potential}

First, after using expression (4) of $S_{0}$, let us write the dynamical equation (20) in following form

$$
\frac{d x}{d t}= \pm \frac{1}{\hbar a W}\left[E-V(x)-\frac{m_{0}^{2} c^{4}}{E-V(x)}\right]\left[\phi_{2}^{2}+\left(a \phi_{1}+b \phi_{2}\right)^{2}\right]
$$

where $W$ represents the wronskian of the function $\phi_{1}$ and $\phi_{2}$. The \pm sign in Eq. (23) indicates that the motion may be in either direction on the $\mathrm{x}$ axis 


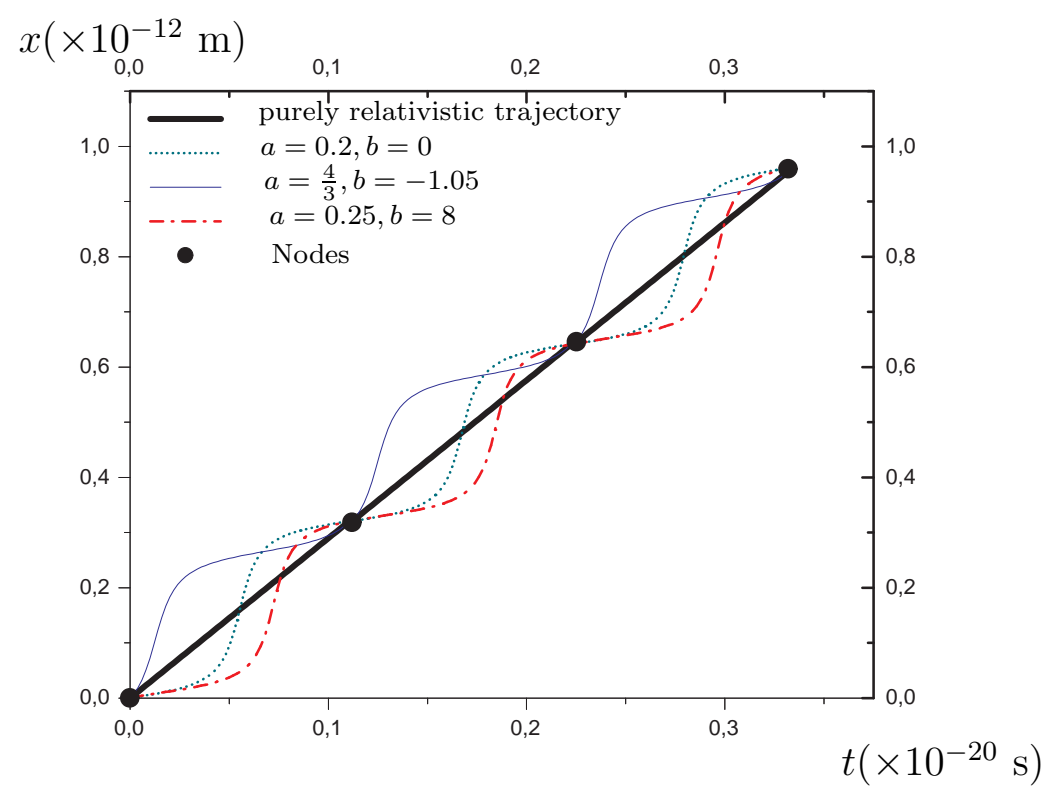

Fig. 1: Relativistic quantum trajectories for an electron of energy $E=\left(2+U_{0}\right)$ Mev. For all the curves, we have chosen $x(t=0)=0$.

[10]. In the case of a massive particle (not a photon) moving under a constant potential $V=U_{0}$, we can distinct two kind of motions, the classically permitted motion $\left(E-U_{0} \ll m_{0} c^{2}\right)$ and the classically forbidden one. First, we review the classically permitted motion. Choosing the two solutions of the Klein-Gordon equation (Eq. (10)) as

$$
\phi_{1}=\sin \left(\frac{\sqrt{\left(E-U_{0}\right)^{2}-m_{0}^{2} c^{4}}}{\hbar c} x\right), \phi_{2}=\cos \left(\frac{\sqrt{\left(E-U_{0}\right)^{2}-m_{0}^{2} c^{4}}}{\hbar c} x\right),
$$

and integrating the dynamical equation (23), we find

$x(t)=\frac{\hbar c}{\sqrt{\left(E-U_{0}\right)^{2}-m_{0}^{2} c^{4}}} \arctan \left[\frac{1}{a} \tan \left(\frac{\left(E-U_{0}\right)^{2}-m_{0}^{2} c^{4}}{\hbar\left(E-U_{0}\right)} t\right)-\frac{b}{a}\right]+x_{0}$. 
This equation represents the time equation of RQTs. As we have mentioned above, $x(t)$ contains four constants since the fundamental equation of motion is a fourth order differential equation. Because the arctangent function is defined on the interval ] $-\pi / 2, \pi / 2[$, Eq. (24) shows that the particle is contained between

$$
-\frac{\hbar c}{\sqrt{\left(E-U_{0}\right)^{2}-m_{0}^{2} c^{4}}} \frac{\pi}{2}+x_{0}
$$

and

$$
\frac{\hbar c}{\sqrt{\left(E-U_{0}\right)^{2}-m_{0}^{2} c^{4}}} \frac{\pi}{2}+x_{0} .
$$

This is not possible. It is necessary to readjust the additive integration constant $x_{0}$ after every interval of time in which the tangent function goes from $-\infty$ to $+\infty$ in such a way to guarantee the continuity of $x(t)$. In this purpose, expression (24) must be rewritten as

$$
\begin{array}{r}
x(t)=\frac{\hbar c}{\sqrt{\left(E-U_{0}\right)^{2}-m_{0}^{2} c^{4}}} \arctan \left[\frac{1}{a} \tan \left(\frac{\left(E-U_{0}\right)^{2}-m_{0}^{2} c^{4}}{\hbar E-U_{0}} t\right)-\frac{b}{a}\right] \\
+\frac{\pi \hbar c}{\sqrt{\left(E-U_{0}\right)^{2}-m_{0}^{2} c^{4}}} n+x_{0} .
\end{array}
$$

with

$$
t \in\left[\frac{\pi \hbar\left(E-U_{0}\right)}{\left(E-U_{0}\right)^{2}-m_{0}^{2} c^{4}}\left(n-\frac{1}{2}\right) ; \frac{\pi \hbar\left(E-U_{0}\right)}{\left(E-U_{0}\right)^{2}-m_{0}^{2} c^{4}}\left(n+\frac{1}{2}\right)\right]
$$

for every integer number $n$. The purely relativistic trajectory is obtained for $a=1$ and $b=0$. Indeed, for these values, Eq. (25) reduces to the relativistic relation

$$
x(t)=\frac{c}{E-U_{0}} \sqrt{\left(E-U_{0}\right)^{2}-m_{0}^{2} c^{4}} t+x_{0} .
$$


In Fig. 1, we have plotted in $(t, x)$ plane for an electron of energy $\left(2+U_{0}\right) M e v$ some trajectories for different values of $a$ and $b$. All these trajectories pass through nodes exactly as we have seen for quantum trajectories of an electron moving under a constant potential [10]. These nodes correspond to the times

$$
t_{n}=\frac{\pi \hbar\left(E-U_{0}\right)}{\left(E-U_{0}\right)^{2}-m_{0}^{2} c^{4}}\left(n+\frac{1}{2}\right) .
$$

The distance between two adjacent nodes is on time axis

$$
\Delta t_{n}=t_{n+1}-t_{n}=\frac{\pi \hbar\left(E-U_{0}\right)}{\left(E-U_{0}\right)^{2}-m_{0}^{2} c^{4}} .
$$

and space axis

$$
\Delta x_{n}=x_{n+1}-x_{n}=\frac{\pi \hbar c}{\sqrt{\left(E-U_{0}\right)^{2}-m_{0}^{2} c^{4}}} .
$$

These distances are both proportional to $\hbar$ meaning that at the classical limit $(\hbar \rightarrow 0)$ the nodes becomes infinitely near, and then, all quantum trajectories tend to the purely relativistic one. As it is explained in Ref. [10, this is the reason why in problems for which $\hbar$ can be disregarded, relativistic quantum trajectories reduce to the purely relativistic one [9, 10]. It is useful to note that at the classical limit $(\hbar \rightarrow 0)$, not only the nodes becomes infinitely near, but in addition the paths of all RQTs go to the path of the purely relativistic one $(a=1, b=0)$. Indeed, let us consider an arbitrary point $P(x, t)$ from any RQT. Obviously, this point is situated between two nodes. Now, if we take the orthogonal projection $P_{0}$ of $P$ on the relativistic trajectory $(a=1$, $b=0)$ and compute the distance $P P_{0}$ we get

$$
P P_{0}=\sqrt{1+\frac{\left(E-U_{0}\right)^{2}}{c^{2}\left[\left(E-U_{0}\right)^{2}-m_{0}^{2} c^{4}\right]}}\left|t_{P}-t_{P_{0}}\right| .
$$




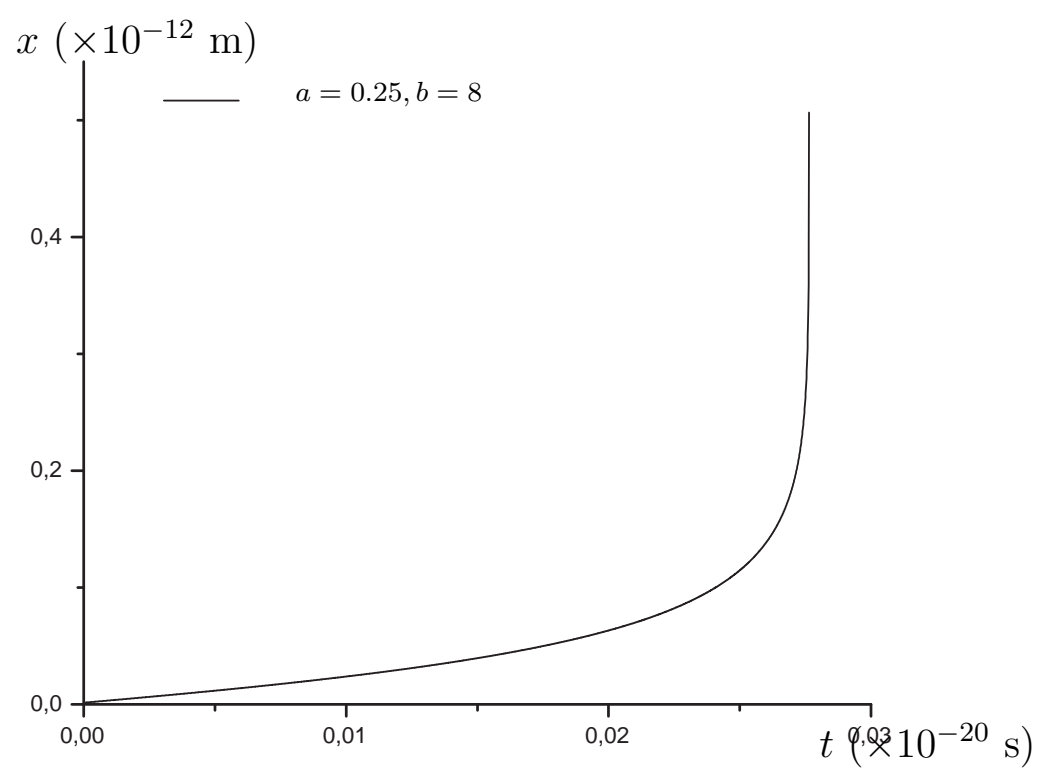

Fig. 2: Relativistic quantum trajectories for an electron of energy $E=$ $\left(U_{0}-2\right)$ Mev moving in the classically forbidden regions. We have chosen $x(t=0)=0$. In the classically forbidden regions, the electron reach quickly an infinite speed.

Since Eq. (23) indicates that $\dot{x}$ is a monotonous function, then, whatever the point $P$ we have $\left|t_{P}-t_{P_{0}}\right|<t_{n+1}-t_{n}$ for every RQT [10]. So, at the classical limit $(\hbar \rightarrow 0)$, because $t_{n+1}-t_{n} \rightarrow 0$, then $\left|t_{P}-t_{P_{0}}\right| \rightarrow 0$ and $P P_{0} \rightarrow 0$. Thus, all RQTs go when $(\hbar \rightarrow 0)$ to the purely relativistic one. This result is in accordance with the fact that our dynamical equations (Eqs (9), (20) and (22)) tend to the relativistic equations when $\hbar \rightarrow 0$.

Now let us consider the classically forbidden motions $\left(E-U_{0}<0\right)$. For this case the solution of Eq. (23) is 


$$
x(t)=\frac{\hbar c}{2 \sqrt{m_{0}^{2} c^{4}-\left(E-U_{0}\right)^{2}}} \ln \left|\frac{1}{a} \tan \left(\frac{m_{0}^{2} c^{4}-\left(E-U_{0}\right)^{2}}{\hbar\left(E-U_{0}\right)} t\right)+\frac{b}{a}\right|+x_{0} .
$$

We see clearly from Eq. (31) that at the finite time

$$
-(2 n+1) \pi \hbar \frac{\left(E-U_{0}\right)}{4\left(\left(E-U_{0}\right)^{2}-m_{0}^{2} c^{4}\right)}
$$

the electron cross an infinite distance and reach an infinite speed. This is in accordance with standard quantum tunneling theories which predict infinite velocities and finite reflexion times for tunneling phenomena (Fletcher [12], Hartman [13]). In Fig. (2), we plotted for an electron of energy $\left(U_{0}-2\right) M e v$ a RQT in the classically forbidden regions with $a=0.25$ and $b=8$. This figure shows clearly how the particle reach an infinite position at a finite time. In particular, in the classically forbidden regions there is no nodes.

\section{The linear potential case}

Here, we investigate the motion of a massive particle (electron) under a potential of the form

$$
V(x)=g x,
$$

for which the Klein-Gordon equation takes the form

$$
-c^{2} \hbar^{2} \frac{\partial^{2} \phi}{\partial x^{2}}+\left[m_{0}^{2} c^{4}-(E-g x)^{2}\right] \phi(x)=0 .
$$

To establish the RQTs for the linear potential case, we integrate Eq. (23), where $\phi_{1}$ and $\phi_{2}$ are, now, two solutions of Eq. (33).

In this paper, we do not present the analytic solutions of Eq. (33), and in order to plot the RQTs, we approach the problem by numeric methods. We, 


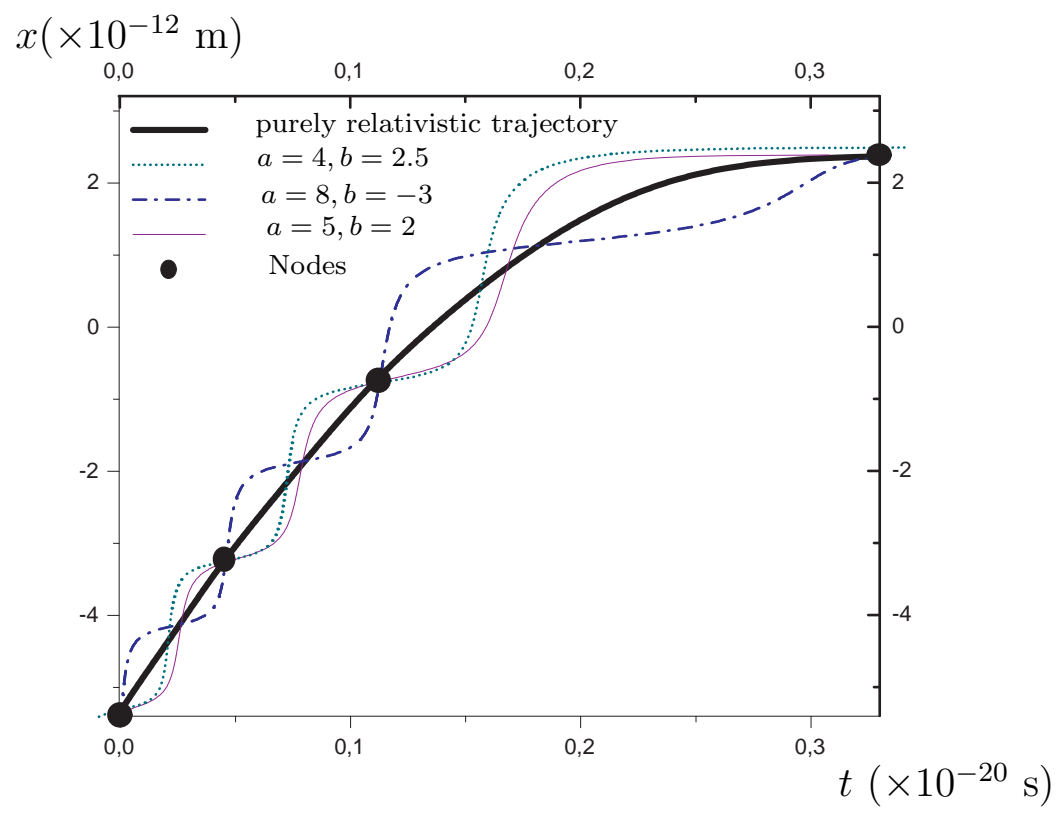

Fig. 3: Relativistic quantum trajectories for an electron of energy $E=2$ Mev moving under a linear potential $(V(x)=$ $g x, g=1$ Kg.m.s.). We have chosen $x(t=0)=-5.4 \times 10^{-12}$ $\mathrm{m}$.

first, integrate numerically Eq. (33) to obtain two independents solutions $\phi_{1}$ and $\phi_{2}$, then, we plot the RQTs from Eq. (23). We opt in the two steps for the Euler integration method. The RQTs for the linear potential are presented in Fig. 3. We choose $E=2 \mathrm{Mev}$, and $g=1 \mathrm{Kg} . \mathrm{m} . \mathrm{s}$. In Ref. [10], we have chosen $g=10^{-9} \mathrm{Kg}$.m.s, which is very small compared with $g=1$ Kg.m.s. We take this last value for relativistic problem to render the quantity $g x$ of the same order as $E$, so that the Klein-Gordon equation do not reduces to the Schrödinger equation.

As we can notice from Fig. 3, the nodes are also present for the linear 
potential case. The distance between two adjacent nodes in Fig. 3 increases as the velocity decreases when it approaches the turning point (point where the velocity vanish). This note will be exposed in Sec. 5. Here we do not investigate the classically forbidden regions. We would like to stress that as for the linear potential in quantum cases, we check that the positions of the nodes on $x$ axis are related to the zeros of the solution of the Schrödinger equation $\phi_{2}$ present in the denominator of the rapport in the expression (4) of the reduced action $S_{0}$. This fact indicates that, the RQTs likes the QTs and it is obvious that the QT are a limit of the RQTs when $c \rightarrow \infty$.

\section{De Broglie's wavelength}

One of the most important ideas that Secs. 3 and 4 bring is the existence of nodes through which all RQTs pass, even the purely relativistic one. In this section, we link the distance between two adjacent nodes to the de Broglie's wavelength

$$
\lambda=\frac{h}{p}
$$

In Eq. (34), $p$ is the relativistic momentum. For a particle moving under a constant potential

$$
p=\frac{\sqrt{\left(E-U_{0}\right)^{2}-m_{0}^{2} c^{4}}}{c} .
$$

By replacing Eq. (35) in Eq. (34), we get

$$
\lambda=\frac{h c}{\sqrt{\left(E-U_{0}\right)^{2}-m_{0}^{2} c^{4}}} .
$$


The distance between two nodes in the case of a constant potential is

$$
\Delta x_{n}=\frac{\pi \hbar c}{\sqrt{\left(E-U_{0}\right)^{2}-m_{0}^{2} c^{4}}} .
$$

From Eqs. (36) and (37) we get

$$
\Delta x_{n}=\frac{\lambda}{2} .
$$

Thus, the de Broglie's wavelength represents the double of the distance between two adjacent nodes. As we have presented in Ref. [10], we can generalize this definition for other potentials. Indeed, if we compute the mean value of $\partial S_{0} / \partial x$ between two adjacent nodes, and taking into account Eqs (25), (28) and (29) we find

$$
\begin{aligned}
\left\langle\frac{\partial S_{0}}{\partial x}\right\rangle \equiv \frac{1}{\Delta x_{n}} \int_{x\left(t_{n}\right)}^{x\left(t_{n+1}\right)} \frac{\partial S_{0}}{\partial x} d x & =\frac{S_{0}\left(x\left(t_{n+1}\right)\right)-S_{0}\left(x\left(t_{n}\right)\right)}{\Delta x_{n}} \\
& =\frac{\sqrt{\left(E-U_{0}\right)^{2}-m_{0}^{2} c^{4}}}{c}
\end{aligned}
$$

which is equal to $p$ (Eq. (35)). We propose to define a new wavelength after substituting $p$ by

$$
p=\left\langle\frac{\partial S_{0}}{\partial x}\right\rangle .
$$

Then for any potential we can write, after using (2)

$$
p=\frac{\pi \hbar}{\Delta x}
$$

with $\Delta x$ is the distance between two adjacent nodes. If we substitute (41) in (34) we find

$$
\Delta x=\frac{\lambda}{2} .
$$


This relation links between the distance separating two adjacent nodes and the de Broglie's wavelength whatever the potential under which the particle moves.

Conclusions To conclude, we would like to stress that we exposed in this article an original approach of the relativistic quantum mechanics. It is a generalization of the one exposed in Refs. 9, 10, 11]. So, we have derived the fundamental relativistic quantum Newton's law expressed into Eqs. (20) and (22). In addition, we have plotted the relativistic quantum trajectories of particles moving under a constant potential and a linear potential in both classically and forbidden regions. For the classically permitted regions, we established the existence of some nodes that we linked successfully to the de Broglie's wavelength.

\section{REFERENCES}

1. A. E. Faraggi and M. Matone, Phys. Lett. B 450, 34 (1999); hep-th/9705108.

2. A. E. Faraggi and M. Matone, Phys. Lett. B 437, 369 (1998); hep-th/9711028.

3. A. E. Faraggi and M. Matone, Int. J. Mod. Phys. A 15, 1869 (2000); hep-th/9809127.

4. G. Bertoldi, A. E. Faraggi and M. Matone, Class. Quant. Grav. 17, 3965 (2000); hep-ph/9909201. 
5. E. R. Floyd, Phys. Rev. D 34, 3246 (1986).

6. E. R. Floyd, Found. Phys. Rev. 9, 489(1996); quant-ph/9707051.

7. E. R. Floyd, Phys. Lett. A 214, 259 (1996);

8. E. R. Floyd, quant-ph/0009070.

9. A. Bouda and T. Djama, Phys. Lett. A 285, 27 (2001); quant-ph/0103071

10. A. Bouda and T. Djama, Physica Scripta 66 (2002) 97; quant-ph/0108022.

11. A. Bouda and T. Djama, Phys. Lett. A 296 (2002) 312-316; quant-ph/0206149.

E. R. Floyd, Phys. Lett. A 296 (2002) 307-311 ; quant-ph/0206114.

12. J. R. Fletcher, J. Phy. C 18 (1985) L55.

13. T. E. Hartman, J. Appl. Phys. 33 (1962) 3427. 